\title{
Selective Unidirectional Saccadic Palsy Due to Cavernous Angioma in the Pontine Tegmentum
}

\author{
Jae-Myung Kim \\ Hak-Loh Lee \\ Jae-Hwan Im \\ Seong-Hyun Min \\ Tai-Seung Nam \\ Seung-Han Lee \\ Department of Neurology, \\ Chonnam National University Hospital, \\ Chonnam National University \\ Medical School, Gwangju, Korea
}

Received October 16, 2018

Revised December 7, 2018

Accepted December 10, 2018

\section{Correspondence}

Seung-Han Lee, MD, PhD

Department of Neurology,

Chonnam National University Hospital,

Chonnam National University

Medical School,

42 Jebong-ro, Dong-gu,

Gwangju 61469, Korea

Tel +82-62-220-6274

Fax +82-62-228-3461

E-mail_nrshlee@chonnam.ac.kr
Dear Editor,

A saccade is a rapid conjugate eye movement that brings the target into the fovea. ${ }^{1}$ Horizontal saccades are generated from burst neurons in the paramedian pontine reticular formation (PPRF) through brainstem network neurons including long-lead burst neurons and omnipause neurons (OPNs). ${ }^{1}$ Clinical disorders including multiple sclerosis, brain tumors, and stroke are known to cause various saccadic impairments. However, in most cases these lesions have not been precisely localized to the PPRF, and they were accompanied by other eye movement abnormalities affecting functions such as smooth pursuit and the vestibuloocular reflex (VOR). ${ }^{2,3}$

A 56-year-old man presented with dizziness and difficulty in looking to the left for 3 days. He did not have hypertension, diabetes mellitus, or other vascular risk factors. A neurological examination revealed impairment of conjugate horizontal saccades to the left in all fields of gaze; that is, saccades from the right gaze to the center as well as from the center to the left were impaired. In addition, he involuntarily blinked whenever attempting a leftward saccade from the right gaze to the center. However, saccades in the contralateral direction and vertical saccades were all spared (Supplementary Video 1 in the online-only Data Supplement). The smooth pursuit and VOR in slow-to-fast head-impulse testing were intact in all directions (Fig. 1), but optokinetic nystagmus $(\mathrm{OKN})$ was poorly evoked by rightward stimulation. Vergence testing was not performed. The visual system was normal, and there was no spontaneous nystagmus. He did not show other neurological impairments such as facial palsy, dysarthria, or motor/sensory dysfunction. Brain MRI revealed a cavernous angioma involving the tegmentum of the left lower pons (Fig. 1). He was referred to the Department of Neurosurgery for gamma-knife surgery, following which his symptoms gradually improved.

Lesions in the pontine tegmentum can cause various ocular motor disorders. ${ }^{1}$ The anatomical proximity allows the simultaneous involvement of nearby structures such as the medial longitudinal fasciculus and abducens nucleus. Therefore, selective horizontal saccadic palsy due to precise PPRF lesions has rarely been reported. ${ }^{4}$

Selective unilateral lesions of the PPRF cause an ipsilateral, conjugate, horizontal saccadic palsy including the fast component of $\mathrm{OKN}$, whereas vergence, vestibular, and pursuit eye movements are typically spared. ${ }^{5}$ Compared to the PPRF, lesions involving the abducens nucleus typically present with 1) loss of all conjugate eye movements toward the lesion, 2) the possibility of the ipsilateral peripheral type of facial palsy, and 3) relative preservation of ipsilaterally directed saccades in the intact hemifield of gaze. If the lesion is restricted to the abducens nucleus, then saccades from the opposite field of gaze to the midline may be present, but they may be slow due to the inhibition of the antagonists being intact. However, inhibition is also affected if the PPRF is extensively involved, and so that saccades directed toward the lesioned side are either absent or extremely slow. ${ }^{1}$

@ This is an Open Access article distributed under the terms of the Creative Commons Attribution Non-Commercial License (https://creativecommons.org/licenses/by-nc/4.0) which permits unrestricted non-commercial use, distribution, and reproduction in any medium, provided the original work is properly cited. 

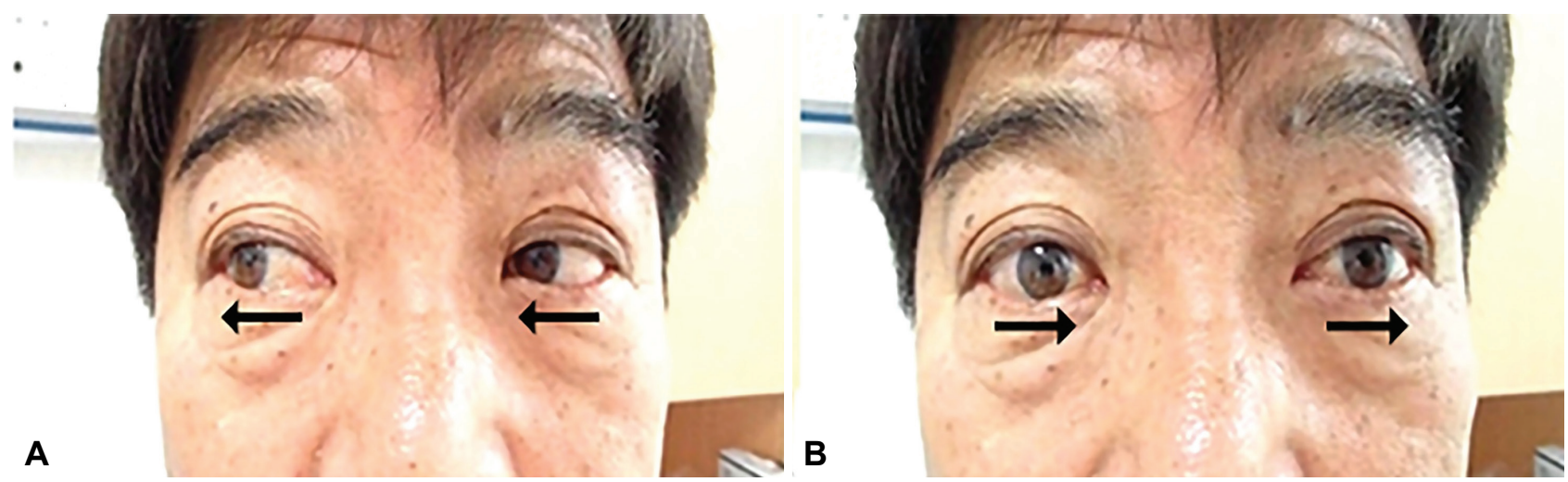

A
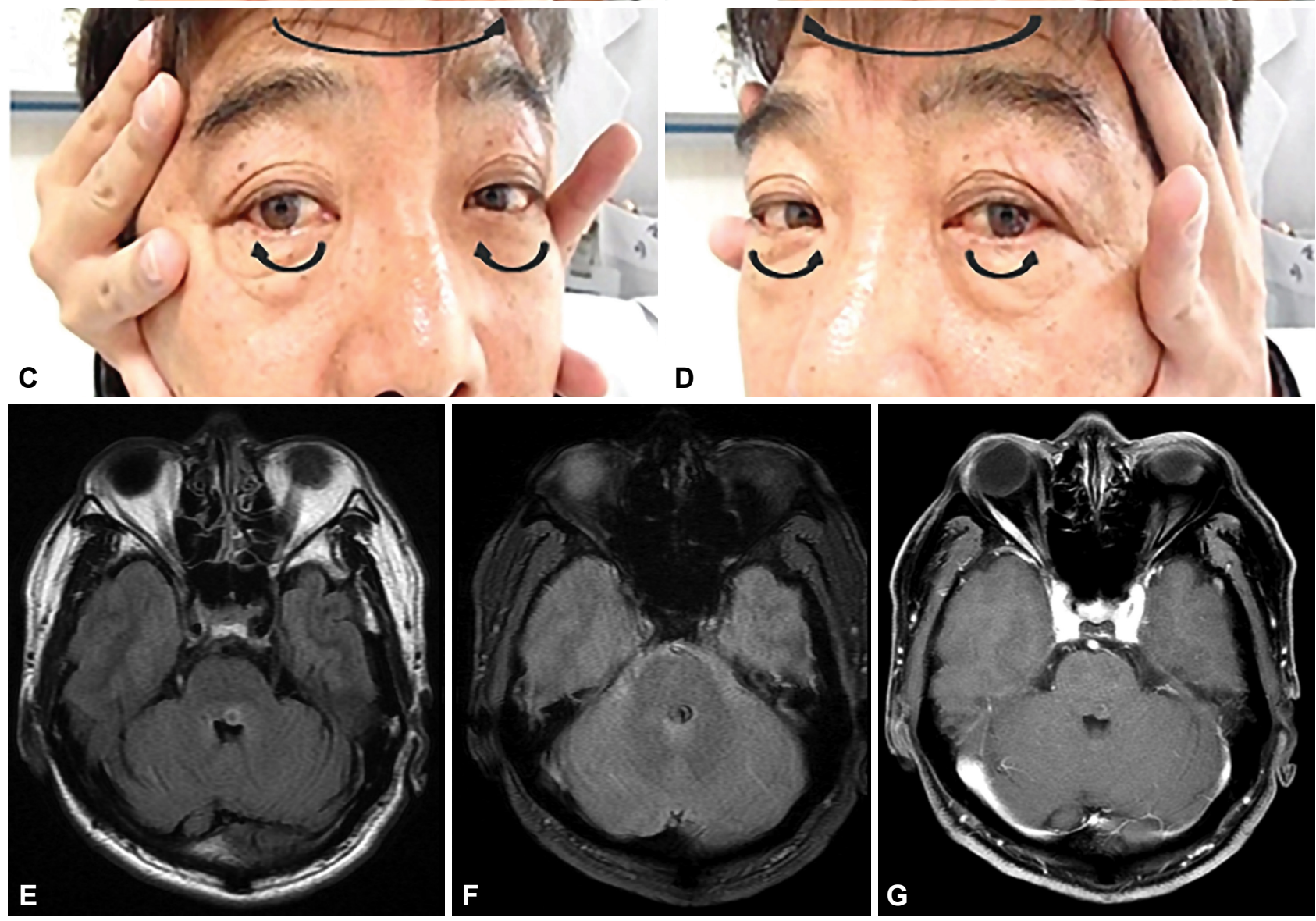

Fig. 1. Ocular motor findings and brain MRI of the patient. Horizontal saccades to the left were impaired $(A, B)$, whereas the low-frequency vestibulo-ocular reflex was spared in both directions (C, D). Brain MRI revealed a 8-mm cavernous angioma with minor hemorrhage involving the tegmentum of left lower pons in FLAIR (E), gradient echo (F), and gadolinium-enhanced T1-weighted (G) images. Direction of attempting a saccade (straight arrows; $A, B)$, direction of head rotation (large curved arrows; C, D), and direction of eye movement (small curved arrows; C, D).

In a previous case of saccadic palsy following cardiac surgery, the patient showed selective saccadic impairment in all directions including vertical plane, even though the lesion was similar to that in our patient. ${ }^{3}$ Even though the patient showed a unilateral lesion in MRI, cardiac surgery usually causes bilateral involvements of the PPRF or more extensive lesions including the OPNs in the nucleus raphe interpositus, which may cause both vertical and horizontal saccades simultaneously. ${ }^{1}$

Our patient exhibited frequent blinking that seemed to represent an attempt to compensate for the impaired saccadic generation. Of particular interest is that he blinked only when attempting a leftward centripetal saccade. Such blinking may cause retraction and rotation of the eye toward a primary position by contraction of several or all of the extraocular muscles. ${ }^{6}$ A literature review revealed that blinking in some patients with saccadic palsy improved the speed of saccades by suppressing the activity of the OPNs. ${ }^{1}$

Unlike previous reports, our case presented the acute and 
complete loss of unidirectional horizontal saccades without other conjugate eye movement disorders. Moreover, the radiological evidence indicated a lesion localized to the PPRF. Various ocular motor disorders (e.g., bilateral horizontal gaze palsy and one-and-a-half syndrome) due to cavernous angiomas in the pontine tegmentum have been reported. ${ }^{1,7}$ However, to our knowledge the selective unilateral involvement of the PPRF in cavernous angioma is extremely rare. The present case shows that cavernous angioma is a possible cause of selective PPRF lesions and thus a possible etiology of saccadic palsy, together with stroke, metastatic tumor, and tuberculoma. ${ }^{1,2,4}$ Detailed bedside neurological examinations of ocular movements are therefore crucial to ensure a correct diagnosis.

\section{Supplementary Video Legend}

Video 1. Saccadic eye movements to the left were impaired in all fields of gaze. More specifically, saccades from the center to the left were not generated at all, and saccades from the right to the center were markedly slow. Note that the patient blinked frequently when attempting a leftward saccade from the right gaze to the center. However, other conjugate eye movements including saccades to the right, the low-frequency (about 0.3 $\mathrm{Hz}$ ) vestibulo-ocular reflex, and smooth pursuit were spared.

\section{Supplementary Materials}

The online-only Data Supplement is available with this article at https://doi.org/10.3988/jcn.2019.15.2.268.
Conflicts of Interest

The authors have no potential conflicts of interest to disclose.

\section{Acknowledgements}

This study was supported by a grant (BCRI18019) of Chonnam National University Hospital Biomedical Research Institute, Gwangju, Korea.

\section{REFERENCES}

1. Leigh RJ, Zee DS. The Neurology of Eye Movements, 5th ed. New York: Oxford University Press, 2015.

2. Saxena R, Menon V, Sinha A, Sharma P, Kumar DA, Sethi H. Pontine tuberculoma presenting with horizontal gaze palsy. J Neuroophthalmol 2006;26:276-278.

3. Eggers SD, Moster ML, Cranmer K. Selective saccadic palsy after cardiac surgery. Neurology 2008;70:318-320.

4. Pham TD, Trobe JD. Selective unidirectional horizontal saccadic paralysis from acute ipsilateral pontine stroke. J Neuroophthalmol 2017; 37:159-161

5. Lee SH, Kim HJ, Kim JS. Ocular motor dysfunction due to brainstem disorders. J Neuroophthalmol 2018;38:393-412.

6. Evinger C, Shaw MD, Peck CK, Manning KA, Baker R. Blinking and associated eye movements in humans, guinea pigs, and rabbits. J Neurophysiol 1984;52:323-339.

7. Washio N, Suzuki Y, Yamaki T, Kase M, Ohtsuka K. Vertical-torsional oscillations and dissociated bilateral horizontal gaze palsy in a patient with a pontine cavernous angioma. J Neurol Neurosurg Psychiatry 2005;76:283-285. 